\title{
Practical Aspects of Volume Control in Chronic Kidney Disease Using Whole Body Bioimpedance
}

\author{
Elizabeth L. Oei ${ }^{\mathrm{a}}$ Stanley L. Fan ${ }^{\mathrm{b}}$ \\ a Department of Renal Medicine and Transplantation, Singapore General Hospital, Singapore, Singapore; \\ ${ }^{b}$ Department of Renal Medicine and Transplantation, Barts Health NHS Trust, London, UK
}

\section{Key Words}

Bioimpedance - Fluid overload - Volume assessment .

Dialysis · Mortality · Fluid management · Clinical outcome

\section{Abstract}

Background: Fluid status is an independent predictor of mortality in dialysis patients. Current methods of fluid assessment have several limitations. Summary: An ideal method should be cheap, portable, easy to perform without extensive training, reproducible and determines patients' excess or deficit of total body water. Bioimpedance analysis (BIA) fulfils many of these criteria and can give additional information on fat and lean tissue composition. The accuracy and precision of BIA has been shown to be equivalent to the 'gold standard' direct estimation techniques. Key Messages: Although there remains some concern about its validity in dialysis patients, fluid overload determined by BIA has been shown to predict mortality. BIA-guided fluid management appears superior to conventional fluid management in achieving clinically important outcomes such as reduction in blood pressure, left ventricular mass index, and arterial stiffness. Accurate setting of dry weight might also help pre- serve residual renal function by limiting episodes of dehydration. Nevertheless, as with all new technologies, there are issues that still need to be resolved. This will be achieved only with larger prospective interventional studies to explore its specific roles in dialysis cohorts.

C) 2015 S. Karger AG, Basel

\section{Can we Improve Fluid Overload and Mortality of Patients on Dialysis Using Bioimpedance Technology?}

Fluid overload (FO) is a major contributor to the high cardiovascular mortality seen in patients with chronic kidney disease and fluid status is an independent predictor of mortality in dialysis patients [1]. However, accurate assessment remains a major challenge. Clinical signs such as oedema, hypertension, and pulmonary congestion do not correlate well to the degree of FO, while classical reference methods based upon dilution techniques, such as those using deuterium or sodium bromide, are invasive, expensive, laborious, and have not been adopted into clinical practice.

\section{KARGER 125}

(C) 2015 S. Karger AG, Basel

$0253-5068 / 15 / 0393-0032 \$ 39.50 / 0$

E-Mail karger@karger.com

www.karger.com/bpu
Dr. Stanley Fan

Department of Renal Medicine and Transplantation

The Royal London Hospital

Whitechapel, London E1 1BB (UK)

E-Mail s.fan@qmul.ac.uk 
There is ongoing interest in bioimpedance technology:

(1) Can it accurately determine fluid status, or more usefully, can it estimate the degree of over- or under-hydration?

(2) Is fluid status determined by bioimpedance an independent predictor of mortality?

(3) Does improvement in bioimpedance hydration measurements correlate with positive clinically important outcomes?

(4) Can bioimpedance technology provide other useful information such as nutritional assessments?

\section{What Are the Different Bioimpedance Analysis Technologies?}

Bioimpedance analysis (BIA) is relatively inexpensive, safe, portable, and gives additional information on the different fluid compartments, fat and lean tissue composition. However, BIA only provides an indirect measure of body composition, and the accuracy of bioimpedance measurements is largely determined by mathematical models and their assumptions, which have generally been validated in Caucasian populations without renal disease.

Single frequency bioimpedance analysis (SFBIA) conventionally utilizes a frequency of $50 \mathrm{kHz}$. This passes through both intracellular fluid (ICF) and extracellular fluid (ECF). Equations to estimate total body water (TBW) are based upon the volume conductor model and multiple regression analysis.

Alternatively, whole body resistance and reactance values derived from SFBIA can be used for bioimpedance vector analysis (BIVA). The combination of the vector length and its direction is defined as the phase angle. Although BIVA has been validated, there is no straightforward relationship between phase angle and any numerical measure of volume. This limits its use in clinical management.

Multi-frequency bioimpedance analysis (MFBIA) devices pass multiple frequencies in the range of $1-1,000$ $\mathrm{kHz}$. The lowest frequencies are used to quantify ECF, as high cell membrane capacitance results in negligible current penetration of ICF. Current at higher frequencies penetrate cell walls, enabling TBW predictions. ICF is then calculated from the difference of the two.

Bioimpedance spectroscopy (BIS) is a more sophisticated model of MFBIA that uses a wide range of frequencies and non-linear mathematical algorithms. BIS offers superior estimations of ECF over SFBIA [2].
Whole body BIS allows the determination of ECF, ICF, and TBW [3], and integration with a physiological model yields hydration status, lean tissue mass, and fat mass [4].

\section{How Can Fluid Overload be Determined?}

The challenge of accurate assessment of fluid status is evidenced by the sheer number of methods that have been developed to address this crucial need. Objective surrogates of fluid status include echocardiography to measure inferior vena cava diameter and left ventricular mass index (LVMI). Measurement of lung comets by ultrasound has also been used to help manage FO. BIA has an advantage over these tests as extensive user training is not required and both inter- and intra-observer variability is low. Moreover, excess fluid in HD patients is predominantly in the ECF compartment. This may explain the superior sensitivity and accuracy of BIA to detect changes in TBW during haemodialysis (HD) ultrafiltration compared to methods that measure vena cava diameter and vena cava collapsibility index [5].

Although biomarkers such as N-terminal fragment of B-type natriuretic peptide (NT-proBNP) have been shown to predict mortality in dialysis patients [6], single measurements of NT-proBNP have not been shown to accurately reflect fluid status [7]. This has been attributed to levels being influenced by both FO and myocardial damage. However, changes in serial measurements of NT-proBNP have been shown to correlate with the changes in TBW measured by BIA [8].

\section{Accuracy of BIA to Detect Clinically Significant Fluid Overload}

To determine the precision of a new test, one normally compares it with the 'gold standard' assessment. In a recent comparison of fluid volume estimates in HD patients by BIA, direct isotopic and dilution methods, the errors in precision and accuracy of BIA was of comparable magnitude to that of conventional gold standard techniques [9]. Moreover, Passauer et al. showed that HD patients who were assessed to be clinically euvolaemic had BIA measurements that were similar to age-matched controls [10].

The accuracy of BIA to diagnose FO can also be 'validated' if it is shown to predict mortality, particularly cardiovascular deaths. Indeed, $2 \mathrm{HD}$ studies confirmed that 
severe FO diagnosed by BIA independently predicted mortality $[11,12]$, with a hazard ratio for all-cause mortality of 2.1 , second only to diabetes [11]. We have also shown that severe FO diagnosed by BIA was an independent predictor of peritoneal dialysis (PD) patient survival (HR 1.83, p < 0.01) [13]. In our PD cohort, the mean FO value of patients that died from cardiac causes was significantly higher than the mean FO value of patients that had 'non-cardiac deaths' (+2.95 vs. $+1.351, \mathrm{p}<0.05$, unpublished data).

An important caveat is that ECF:TBW ratio is affected by both abnormal hydration and muscle wasting. Thus, FO determined by BIA can be confounded if patients have marked Protein Energy Wasting (PEW). BIA may be predicting increased mortality by detecting patients with PEW and Malnutrition Inflammation Atherosclerosis syndrome rather than accurately identifying patients with the detrimental effects caused by FO.

\section{Can BIA Help Determine Dry Weight?}

Determination of dry weight (DW) has historically been based on trial and error. However, reducing target DW until hypotension (either symptomatic or asymptomatic) ensues cannot be recommended. For example, this method would be both insensitive and dangerous in cardiac failure patients. Technologies such as echocardiography and biochemical biomarkers may help identify patients that are FO, but BIA is unique in estimating the volume of FO. However, BIA values must be taken in a clinical context and BIA cannot determine how or if FO can be corrected safely (e.g., in patient with cardiac failure). BIA does not discriminate between intravascular and interstitial ECF excess. A study of hypoalbuminaemic PD patients showed FO by BIS was not associated with an increased plasma volume and attempts to normalise the ECF:TBW ratio in this setting may lead to intravascular hypovolaemia and hypotension [14]. Hence, it has been suggested that trends rather than absolute BIA measurements may be more meaningful; changes in hydration status measured by BIA correlated well with isotopic methods [15].

Despite the above concerns, the use of BIA to set ideal DW in HD was shown to lower blood pressure (BP) without increasing intradialytic adverse events [16]. In a prospective study of $55 \mathrm{HD}$ patients, BIA directed DW reduction over 3 months achieved a $9.9 \mathrm{~mm} \mathrm{Hg}$ drop in predialysis systolic BP for every litre of FO correction $(\mathrm{r}=0.55, \mathrm{p}<0.001)[17]$.
Two recent randomised controlled trials have shown that ultrafiltration (UF) guided by BIA achieved superior outcomes when compared to patients whose UF were guided by conventional strategies. Hur et al. showed that UF guided by BIS achieved a significant decrease in timeaveraged FO (TAFO) over 1 year, leading to decreased LVMI (131 \pm 36 to $\left.116 \pm 29 \mathrm{~g} / \mathrm{m}^{2}, \mathrm{p}<0.001\right)$, arterial stiffness (pulse wave velocity $8.7 \pm 2.8$ to $8.1 \pm 2.3 \mathrm{~m} / \mathrm{s}, \mathrm{p}=$ 0.009 ), BP (predialysis SBP $129 \pm 17$ to $120 \pm 19$, predialysis DBP $76 \pm 7$ to $73 \pm 9, p<0.001$ ), and the use of antihypertensives ( 23 to $11 \%, \mathrm{p}=0.008$ ), when compared to control patients [18]. Meanwhile Onofriescu et al. have gone a step further to show lower mortality of patients randomised to have UF guided by BIS. Unadjusted hazard ratio for all-cause mortality in the BIA group was 0.100 (95\% confidence interval $0.013-0.805, \mathrm{p}=0.03$ ) [19].

It should be acknowledged that the interventional group $(\mathrm{n}=78)$ in the study by Hur et al. developed significantly decreased urine output and an increased proportion developed anuria. However, loss of residual renal function (RRF) may be a reflection of the rate of FO correction rather than inaccuracy of BIA to predict accurate DW. It is reassuring that in a population of $237 \mathrm{PD}$ patients, correction of FO did not predict the rate of loss of RRF [20]. Moreover, it has been postulated that BIA may be particularly helpful to prevent hypertensive patients on dialysis becoming dehydrated and developing acute or chronic loss of RRF [16].

\section{Using Bioimpedance for Managing Fluid Status in PD Patients}

Compared to HD patients, $\mathrm{PD}$ patients are seen infrequently. Minor adjustments of DW based on response to fluid removal are therefore not possible in $\mathrm{PD}$, thereby increasing the need for technologies that accurately assesses the degree of FO. There are, however, conflicting reports on whether the presence of peritoneal dialysate in the abdominal cavity significantly alters BIA estimation of FO. As resistance to flow of electrical currents is predominantly in the limbs, it has been assumed that the effects of peritoneal dialysate would not be clinically relevant. Many centres perform BIA with peritoneal dialysate instilled, as this is quicker and more convenient to both patients and staff.

Two studies using the Body Composition Monitor (BCM, Fresenius Medical Care, Bad Homberg) did not find that the absence or presence of peritoneal dialysate 
significantly altered ECF, ICF, or overhydration measurements [21, 22]. However, there was better correlation between cardiac echography indices of overhydration and BIS measurements done while the abdomen was empty [22]. Using the 8-tactile electrode system (InBody 720 , Biospace, Korea), draining out dialysate caused an increase in truncal resistance and reactance. Measurements performed with peritoneal dialysate in-situ resulted in higher estimations of muscle, fat, and bone mineral content. Using higher dextrose concentrations in the peritoneal dialysis solution may exacerbate differences in measurements between an empty and full peritoneal cavity [23].

Despite the potential technical issues about how measurements should be obtained from PD patients, improved clinical outcomes were found in the studies using this technology. We found that patients showing decrements in FO measured by BIA had corresponding decrements in circulating cardiac Tropinin-T levels (personal communication). In the study by Luo et al., patients and their clinicians were randomly allocated to be informed or blinded to BIA results. The group that were given the results achieved a significant reduction of $\mathrm{OH}(2.3 \pm 2.0$ to $1.7 \pm 1.51, \mathrm{p}<0.05)$ over 3 months, while there was an increase in $\mathrm{OH}$ in the blinded group $(2.2 \pm 1.7$ to $2.5 \pm$ $1.8 \mathrm{l}, \mathrm{p}<0.05)$ [24]. In addition, the intervention group achieved lower BP (systolic $137 \pm 19$ to $133 \pm 19, \mathrm{p}<0.05$; diastolic $81 \pm 15$ to $78 \pm 12, \mathrm{p}<0.05)$, without change in RRF.

\section{BIS Can be Particularly Useful to Preserve RRF}

While it is true that aggressive volume control can accelerate loss of RRF, there is no data showing that hypervolaemia is any better than euvolaemia in preserving RRF. In fact, a retrospective study found that overhydration was associated with loss of RRF [25], but increments and decrements of hydration status measured by BIS were not associated with preservation or reduction of RRF [20].

The true challenge is not straying from the fine line that divides the deleterious effects of FO and dehydration. This is perhaps where BIS offers a unique advantage in giving a precise numerical value for overhydration.

Strict salt and fluid restriction can successfully control $\mathrm{BP}$ in PD patients, but this can be at a significant cost to $\mathrm{RRF}$ [26], possibly because a proportion of patients in this study became hypovolaemic. It is therefore of note that we found that BIS allowed us to identify a cohort of se-

Volume Control in CKD Using Whole

Body Bioimpedance verely overhydrated patients where volume control permitted a $50 \%$ reduction in the use of antihypertensive medication without inducing symptoms of dehydration or accelerated loss of RRF [27].

\section{Directions for the Future}

Although there is convincing evidence that BIS can improve fluid management, it is essential that we obtain better data to support BIA technology in clinical use. Cross-sectional observational studies in both $\mathrm{HD}$ and $\mathrm{PD}$ suggest a large proportion of dialysis patients would be defined as overhydrated by BIA $[28,29]$. To undertake wholesale changes to clinical practice is perhaps premature, as harm can be inflicted if BIA is inappropriately interpreted.

Different devices use different equations to predict ECF and ICF, resulting in wide limits of variation, even when measured in the same individual. Additionally, impedance and specific resistance of the body are dependent on electrolyte concentrations and on the percentage of red blood cells, due to the insulating properties of cell membranes. Both of these are frequently abnormal in dialysis patients, and these factors have led to uncertainties in the use of normative fluid volumes developed in nondialysis populations.

The current limitations of accuracy and precision of 'Whole Body' BIS prevents its use in real-time management of intradialytic hypotension in HD. However, a segmental approach using calf BIS based on flattening of continuous resistance curves is emerging as a method of identifying attainment of UF target, as opposed to the direct determination of DW [30].

An additional advantage of BIA is the provision of clinically significant nutritional indices. We have confirmed that patients defined to be malnourished by Subjective Global Assessments had reduced lean tissue mass (LTM) and fat tissue mass (FTM). Patients with very low LTM (normalised for age/gender) also had increased mortality. Subsequent loss of LTM monitored by BIA enhanced the ability to predict mortality (Abstract Reference To Follow - Accepted for ISPD Meeting, Madrid 2014).

\section{Conclusion}

Fluid status is critically important for the management of dialysis patients but its accurate assessment remains challenging. An ideal method should determine TBW and estimate the deviation from optimum fluid volume 
content of the individual. Despite limitations, BIA technology purports to provide this information in a relatively cheap, portable, and easy-to-use machine. FO estimated by BIA has been shown to predict mortality. Moreover, evidence to date suggests that BIA-guided fluid management can lead to clinical improvements that we traditionally value - BP, reduction of intradialytic hypotension, decreased LVMI, and arterial stiffness. There is also circumstantial evidence to suggest that BIA may have an important role in preserving RRF.

As with all new technologies, there are issues that still need to be resolved. This will be achieved only with larger prospective interventional studies to explore its specific roles in dialysis cohorts.

\section{References}

$>1$ Ozkahya $M$, et al: Long-term survival rates in haemodialysis patients treated with strict volume control. Nephrol Dial Transplant 2006; 21:3506-3513.

$>2$ Jaffrin MY, Morel H: Body fluid volumes measurements by impedance: a review of bioimpedance spectroscopy (BIS) and bioimpedance analysis (BIA) methods. Med Eng Phys 2008;30:1257-1269.

3 Moissl UM, et al: Body fluid volume determination via body composition spectroscopy in health and disease. Physiol Meas 2006;27: 921-933.

$\checkmark 4$ Chamney PW, et al: A whole-body model to distinguish excess fluid from the hydration of major body tissues. Am J Clin Nutr 2007;85: 80-89.

5 Kraemer M: A new model for the determination of fluid status and body composition from bioimpedance measurements. Physiol Meas 2006;27:901-919.

$\checkmark 6$ Paniagua R, et al: NT-proBNP, fluid volume overload and dialysis modality are independent predictors of mortality in ESRD patients. Nephrol Dial Transplant 2010;25:551-557.

7 Agarwal R: B-type natriuretic peptide is not a volume marker among patients on hemodialysis. Nephrol Dial Transplant 2013;28: 3082-3089.

$>8$ Davenport A: Changes in N-terminal probrain natriuretic peptide correlate with fluid volume changes assessed by bioimpedance in peritoneal dialysis patients. Am J Nephrol 2012;36:371-376.

$>9$ Raimann JG, et al: Comparison of fluid volume estimates in chronic hemodialysis patients by bioimpedance, direct isotopic, and dilution methods. Kidney Int 2014;85:898908.

10 Passauer J, et al: Evaluation of clinical dry weight assessment in haemodialysis patients using bioimpedance spectroscopy: a crosssectional study. Nephrol Dial Transplant 2010;25:545-551.
11 Wizemann $\mathrm{V}$, et al: The mortality risk of overhydration in haemodialysis patients. Nephrol Dial Transplant 2009;24:1574-1579.

12 Chazot C, et al: Importance of normohydration for the long-term survival of haemodialysis patients. Nephrol Dial Transplant 2012;27: 2404-2410.

13 O'Lone EL, et al: Clinical significance of multi-frequency bioimpedance spectroscopy in peritoneal dialysis patients: independent predictor of patient survival. Nephrol Dial Transplant 2014;29:1430-1437.

14 John B, et al: Plasma volume, albumin, and fluid status in peritoneal dialysis patients. Clin J Am Soc Nephrol 2010;5:1463-1470.

15 Davies SJ, et al: Longitudinal relationships between fluid status, inflammation, urine volume and plasma metabolites of icodextrin in patients randomized to glucose or icodextrin for the long exchange. Nephrol Dial Transplant 2008;23:2982-2988.

16 Machek P, et al: Guided optimization of fluid status in haemodialysis patients. Nephrol Dial Transplant 2010;25:538-544.

17 Moissl U, et al: Bioimpedance-guided fluid management in hemodialysis patients. Clin J Am Soc Nephrol 2013;8:1575-1582.

18 Hur E, et al: Effect of fluid management guided by bioimpedance spectroscopy on cardiovascular parameters in hemodialysis patients: a randomized controlled trial. Am J Kidney Dis 2013;61:957-965.

19 Onofriescu M, et al: Bioimpedance-guided fluid management in maintenance hemodialysis: a pilot randomized controlled trial. Am J Kidney Dis 2014;64:111-118.
20 McCafferty K, Fan S, Davenport A: Extracellular volume expansion, measured by multifrequency bioimpedance, does not help preserve residual renal function in peritoneal dialysis patients. Kidney Int 2014;85:151-157.

21 Parmentier SP, et al: Influence of peritoneal dialysis solution on measurements of fluid status by bioimpedance spectroscopy. Int Urol Nephrol 2013;45:229-232.

22 Sipahi S, et al: Body composition monitor measurement technique for the detection of volume status in peritoneal dialysis patients: the effect of abdominal fullness. Int Urol Nephrol 2011;43:1195-1199.

23 Davenport A: Does peritoneal dialysate affect body composition assessments using multifrequency bioimpedance in peritoneal dialysis patients? Eur J Clin Nutr 2013;67:223225.

24 Luo YJ, et al: Volume control in peritoneal dialysis patients guided by bioimpedance spectroscopy assessment. Blood Purif 2011;31: 296-302.

25 Fan S, Sayed RH, Davenport A: Extracellular volume expansion in peritoneal dialysis patients. Int J Artif Organs 2012;35:338-345.

26 Gunal AI, et al: Strict volume control normalizes hypertension in peritoneal dialysis patients. Am J Kidney Dis 2001;37:588-593.

-27 Burke SE, Fan SL: Clinical experience using bioimpedance to optimize blood pressure control. Perit Dial Int 2013;33:205-208.

28 Hur E, et al: Bioimpedance and echocardiography used interchangeably in volume comparison of dialysis patients. Hippokratia 2012; 16:329-334.

29 Van Biesen W, et al: Fluid status in peritoneal dialysis patients: the European Body Composition Monitoring (EuroBCM) study cohort. PLoS One 2011;6:e17148.

$>30$ Liu L, et al: Determination of fluid status in haemodialysis patients with whole body and calf bioimpedance techniques. Nephrology (Carlton) 2012;17:131-140. 